\title{
Factores que influyen en la duración de la lactancia materna en las estudiantes universitarias $^{1}$
}

\author{
María José Alpízar Campos², Jennifer Canales Madrigal ${ }^{3}$, Rodolfo Darío Moreira Álvarez ${ }^{4}$, Marisol Castillo \\ Ramírez
}

Institución: Universidad de Costa Rica, Sede de Occidente

\section{RESUMEN}

Las estudiantes universitarias que amamantan enfrentan retos en relación con el complimiento de diferentes roles, motivo por el que este estudio trató de determinar los factores que influyen en la duración de la lactancia materna en esta población. Este artículo corresponde a una investigación cualitativa de tipo descriptivo, con una población de diez estudiantes universitarias que amamantaban a sus hijos e hijas. Para la recolección de datos se utilizó la entrevista semiestructurada y el análisis se realizó triangulando los datos obtenidos, la teoría de I. King y los aportes de los investigadores. Los resultados señalaron que las estudiantes universitarias perciben que la lactancia materna es un derecho, un beneficio, y el mejor alimento que pueden brindarle a sus hijos e hijas. La familia o amigos generalmente son los que plantean la idea de abandonar la lactancia materna, además el entorno familiar influye directamente en la duración de la lactancia materna. Se concluye que el éxito en la lactancia de las estudiantes universitarias madres va estar influenciado por diferentes factores tantos personales, interpersonales y sociales que son dinámicos y que se relacionan entre sí, además de las redes de apoyo, educación en salud, acompañamiento y la acciones en pro de la lactancia materna de la institución universitaria y de sus propios entornos familiares.

Palabras clave: Enfermería; Lactancia-Materna; Amamantamiento.

DOI 10.15517/revenf.v0iNo. 37.34905

${ }^{1}$ Fecha de recepción: 17 de Octubre 2018

${ }^{2}$ Enfermera. Universidad de Costa Rica, Sede de Occidente. San Ramón, Costa Rica. Correo electrónico: majo.alpizar@gmail.com

${ }^{3}$ Enfermera. Universidad de Costa Rica, Sede de Occidente. San Ramón, Costa Rica. Correo electrónico: nee-cm14@hotmail.com

${ }^{4}$ Enfermero. Universidad de Costa Rica, Sede de Occidente. San Ramón, Costa Rica. Correo electrónico: dario-u@hotmail.com

${ }^{5}$ Enfermera. Máster en Pediatría con énfasis en niñez y adolescencia. Docente Universidad de Costa Rica, Sede de Occidente.

San Ramón, Costa Rica. Correo electrónico: marisol.castillo@ucr.ac.cr 


\title{
Factors that influence the duration of breastfeeding in university students ${ }^{1}$
}

\author{
María José Alpízar Campos², Jennifer Canales Madrigal ${ }^{3}$, Rodolfo Darío Moreira Álvarez ${ }^{4}$, Marisol Castillo \\ Ramírez
}

Institution: University of Costa Rica, Headquarters of the West

\begin{abstract}
The breastfeeding university students face challenges concerning the fulfillment of different roles, this is how this study tried to determine which factors influence the duration of breastfeeding in this population. This article corresponds to descriptive qualitative research, with a population of ten university students who breast-fed their sons and daughters. For data collection, the semi-structured interview was used and triangulating the data obtained, the theory of I. King and the contributions of the researchers. The results indicated that university students perceive that breastfeeding is a right, a benefit, and the best food they can provide to their children. The family or friends are usually those who raise the idea of abandoning breastfeeding, also, the family environment directly influences the duration of breastfeeding. It is concluded that the success in breastfeeding of university students mothers will be influenced by different personal, interpersonal and social factors that are dynamic and related to each other, besides, to support networks, health education, accompaniment and actions in favor of breastfeeding the university institution and their family environments.
\end{abstract}

Keywords: Breastfeeding; Brestmilk; Nursing.

DOI 10.15517/revenf.v0iNo. 37.34905

${ }^{1}$ Date of receipt: October 17,2018

Date of acceptance: June 25, 2019

${ }^{2}$ Nursing. University of Costa Rica, Headquartes of the West. San Ramón, Costa Rica. E-mail: majo.alpizar@gmail.com

${ }^{3}$ Nursing. University of Costa Rica, Headquartes of the West. San Ramón, Costa Rica. E-mail: nee-cm14@hotmail.com

${ }^{4}$ Nursing. University of Costa Rica, Headquartes of the West. San Ramón, Costa Rica. E-mail: dario-u@hotmail.com

${ }^{5}$ Nursing. Master in Pediatrics with emphasis on childhood and adolescence. Professor University of Costa Rica, Headquartes of the West. San Ramón, Costa Rica. E-mail: marisol.castillo@ucr.ac.cr 


\title{
Fatores que influenciam a duração da amamentação em estudantes universitarios ${ }^{1}$
}

\author{
María José Alpízar Campos², Jennifer Canales Madrigal ${ }^{3}$, Rodolfo Darío Moreira Álvarez ${ }^{4}$, Marisol Castillo \\ Ramírez
}

Instituição: Universidade de Costa Rica, Sede do Ocidente

\section{RESUMO}

Os estudantes universitários que amamentam enfrentam desafios em relação à realização de diferentes papéis, assim como este estudo procurou determinar quais fatores influenciam a duração do aleitamento materno nessa população. Corresponde a uma pesquisa qualitativa, do tipo descritiva, contou-se com uma população de dez universitários que amamentavam seus filhos e filhas. Para coleta de dados, utilizou-se a entrevista semiestruturada e a análise foi realizada com base na triangulação dos dados obtidos, a teoria de I. King e as contribuições dos pesquisadores. Os universitários percebem que o aleitamento materno é um direito, um benefício e a melhor alimentação que podem proporcionar aos seus filhos. Família ou amigos são geralmente aqueles que levantam a ideia de abandonar a amamentação, além do ambiente familiar influenciar diretamente a duração da amamentação. Conclui-se que o sucesso da amamentação de mulheres universitárias será influenciado por diferentes fatores pessoais, interpessoais e sociais, dinâmicos e relacionados entre si, além de redes de apoio, educação em saúde, acompanhamento e ações em prol da maternidade. do aleitamento materno da instituição universitária e do próprio ambiente familiar.

Palavras-chave: Aleitamento-Materno; Amamentação; Enfermagem.

DOI 10.15517/revenf.v0iNo. 37.34905

${ }^{1}$ Data de recepção: 17 de Outubro de 2018

Data de aceitação: 25 de Junho de 2019

${ }^{2}$ Enfermeira. Universidade de Costa Rica, Sede do Ocidente. San Ramón, Costa Rica. Correio eletrônico: majo.alpizar@gmail.com

${ }^{3}$ Enfermeira. Universidade de Costa Rica, Sede do Ocidente. San Ramón, Costa Rica. Correio eletrônico: nee-cm14@hotmail.com

${ }^{4}$ Enfermeiro. Universidade de Costa Rica, Sede do Ocidente. San Ramón, Costa Rica. Correio eletrônico: dario-u@hotmail.com

${ }^{5}$ Enfermeira. Mestrado em Pediatria com ênfase na infância e adolescência. Professor na Universidade de Costa Rica, Sede do Ocidente.

San Ramón, Costa Rica. Correio eletrônico: marisol.castillo@ucr.ac.cr 


\section{INTRODUCCIÓN}

Como proceso, la lactancia materna involucra brindar la alimentación al hijo o hija por medio de la leche materna, lo cual no solo implica aspectos fisiológicos, sino que va más allá involucrando, dado que incluye el entorno en el que se desarrolla ${ }^{1}$; por tanto, es un proceso complejo que, para algunas mujeres, se convierte en un reto al desempeñar el rol de madres y estudiantes universitarias, siendo el segundo uno de los más determinantes para tomar la decisión de amamantar o no a sus hijos, al punto de que marca un antes y un después en sus planteamientos respecto de su formación, sobre todo en aquellas mujeres que se enfrentaron a este hecho en un periodo temprano de su desarrollo humano, por lo que cambiaron así las expectativas y presiones en cuanto a su formación y profesión ${ }^{2}$.

Además, Castañeda ${ }^{2}$ menciona que una estudiante universitaria que se convierte en madre y se encara nuevos retos, debe replantearse su rol de estudiante ya que, si este no interfiere con la demanda de tiempos de cuidado que la maternidad trae consigo, podría priorizar la maternidad; por consiguiente, disminuiría su carga académica o pausaría los estudios para compatibilizar ambas cosas.

Tal como se expone, como parte de la maternidad, la lactancia conlleva enfrentar diferentes situaciones que sobrepasan el aspecto fisiológico; por ende, muchas veces no termina de forma exitosa.

B1.En torno a la lactancia materna, son muchos sus beneficios, entre ellos, la cantidad de nutrientes de calidad u de cantidad adecuada, los cuales contribuyen al desarrollo físico y mental del infante, además de que se establece un vínculo entre el recién nacido y su madre al momento de amamantamiento ${ }^{3}$ : partiendo de lo mencionado, hasta el momento, no se conoce ningún otro alimento que se compare con la leche materna, por lo que mucha de la evidencia científica la recomienda como alimento exclusivo para menores de seis meses ${ }^{4}$. De igual modo, se busca incentivar que el periodo de lactancia materna no tenga una duración específica o determinada, por lo que se recomienda eliminar el adjetivo en "lactancia prolongada", ya que esta la decisión de finalizarla corresponde a la madre y al infante, y estará condicionada por las experiencias y oportunidades con las que se cuenten ${ }^{5}$.

Por su parte la Organización Mundial de la Salud (OMS) y el Fondo de las Naciones Unidas para la Infancia (UNICEF por sus siglas en inglés) ${ }^{6}$ velan de manera constante por el desarrollo de estrategias encaminadas al fomento de la lactancia materna en los diferentes escenarios sociales, pues es la mejor estrategia y de más bajo costo para reducir la morbimortalidad infantil, razón por la que, para los agentes de salud y población con conocimiento en el tema, el promover y apoyar la lactancia materna garantiza no solo un medio de promoción de la salud, sino que se constituye en un tema de interés para la salud pública que utiliza la atención primaria y educación como estrategia ${ }^{6}$.

En términos porcentuales, la OMS señala que al menos el $45 \%$ de las defunciones infantiles se deben a la desnutrición y que, si todos los infantes de los 0 a 23 meses de edad fueran amamantados, anualmente se podrían salvar aproximadamente 800000 mil menores de cinco años ${ }^{7}$ Al respecto, la UNICEF afirma que amamantar a los bebés en la primera hora de nacidos puede reducir en $22 \%$ las muertes neonatales y aumentar en seis veces la supervivencia de los niños amamantados en comparación con los que no recibieron lactancia materna ${ }^{7}$ Lo anterior significa que los lactantes alimentados exclusivamente con leche materna tienen solo el $12 \%$ del riesgo de 
muerte comparado con aquellos que no fueron amamantados, además entre los menores de seis meses que no la recibieron aumentó la mortalidad de 3-5 veces, en varones, y de 4-1 veces, en niñas, al compararlos con quienes sí la recibieron: tales resultados son apoyados por estudios en niños de 6-23 meses, en quienes cualquier forma de lactancia materna se asoció con una reducción del $50 \%$ en las muertes ${ }^{8}$.

También en relación con las sus beneficios, la lactancia materna protege contra la diarrea y las infecciones respiratorias también esta evitaría aproximadamente la mitad de todos los episodios de diarrea y un tercio de las infecciones respiratorias, sin dejar de lado que -por periodos más largos- se asocia con una reducción del $26 \%$ en las probabilidades de sobrepeso u obesidad, y del 19\% en la incidencia de leucemia infantil, además de que se relaciona consistentemente con un mayor rendimiento en pruebas de inteligencia en niños y adolescentes ${ }^{8}$. Por último, también se registra ventajas para la madre en cuanto a disminuir el cáncer de ovario en un $30 \%$, vinculado con periodos más largos de lactancia ${ }^{8}$, así como una asociación inversa entre la lactancia materna y el cáncer de mama.

Considerando lo mencionado, desde la enfermería es necesaria la intervención en un tema tan relevante, a partir de una visión interactiva que brinde una atención integral y aborde a la persona como un todo, razón por la en este trabajo se planteó como objetivo analizar los factores personales, interpersonales y sociales que influyen en la duración de la lactancia materna en las estudiantes universitarias que amamantan a sus infantes, debido a que conocer al respecto permite guiar mejor el proceso de atención y poner en marcha estrategias dirigidas a los aspectos claves que ayudarían a las estudiantes madres que amamantan.

\section{MATERIALES Y MÉTODOS}

La presente investigación es cualitativa de tipo descriptivo, ya que "los estudios descriptivos son aquellos que buscan especificar las propiedades de las personas, grupos, comunidades, objetos o cualquier otra unidad sometida a la investigación", para cuyo diseño se debe considerar tres aspectos fundamentales: el tiempo, la fuente de los datos y la amplitud del foco 9 .

En la mayoría de los estudios, es necesario tomar una muestra; sin embargo, en este no fue necesario, ya que se abordó la totalidad de la población, formada por 10 estudiantes madres que amamantan, matriculadas en la Universidad de Costa Rica, sede de Occidente, recinto de San Ramón en el periodo en el que se desarrolló la investigación.

Los criterios de selección específicos para la investigación fueron: mujeres madres en condición de estudiantes que se encontrarán amamantando a sus infantes en la Universidad de Costa Rica, sede de Occidente, recinto San Ramón durante el segundo semestre del 2017, que aceptaran voluntariamente participar en la investigación, que se mantuvieran durante el desarrollo de esta y que hubieran firmado el consentimiento informado.

Para la recolección de datos se utilizó la entrevista semiestructurada -la cual es más íntima, flexible y abierta $^{10}$ elaborada por los investigadores con la finalidad de obtener la información necesaria para lograr los objetivos de la investigación. Antes de aplicarla, fue validada por medio del juicio de expertos los cuales aportaron recomendaciones para mejorarla; el entrevistador visitó a cada participante en su respectiva casa de 
habitación o en las instalaciones de la Universidad de Costa Rica, sede de Occidente, recinto San Ramón, y escribió las respuestas en primera persona.

En relación con el contacto con las participantes, se hizo por medio de llamadas telefónicas desde las que se agendó una cita para realizar la entrevista conforme a la disposición y tiempo de cada participante. Todas las entrevistas se realizaron durante el mes de octubre del 2017: estas no fueron grabadas, ya que se contó con el tiempo suficiente para plasmar de manera completa la información.

Respecto del instrumento, se hizo considerando que la organización y división de las temáticas son esenciales para el análisis de datos y utilizando la categorización apriorística, la cual establece la construcción de categorías y subcategorías antes del proceso de recolección de datos ${ }^{11}$.

Al finalizar la recolección de la información, se utilizó el programa de análisis cualitativo Atlas.ti@, en su versión 8.0: este tipo de programas automáticamente dejan un margen derecho para los códigos y los anotan, por tanto, permite categorizar las respuestas en códigos y brindar un panorama más concreto para elaborar el análisis. Antes del análisis, se transcribió la información o datos obtenidos: al seleccionar la pregunta que se categorizó con el programa Atlas.ti $\subset$, era necesario elaborar documentos de Word, en el que se colocó las respuestas tal y como las mencionaron o seleccionaron las participantes. Seguidamente, se agrupó los documentos de Word en una base de datos para incluir todas las respuestas: una vez agrupadas, fueron exportadas al documento asignarles un código, el cual fue creado en el programa y que respondió a los conceptos de los sistemas personal, interpersonal y social de la teoría de consecución de objetivos.

Una vez exportada, la base de datos se organizó y se asignó un código a cada respuesta; luego, se hizo "un barrido" o revisión de los datos donde: 1 . Se percató que la información captada representaba el pensar de las participantes, al encontrarse una congruencia entre la codificación y el pensar de la población entrevistada. 2. Al utilizar la teoría de Imogene King, la cual incluye conceptos definidos y estructurado en sistemas, se logra incluir las categorías más relevantes en las respuestas, de modo que se dé congruencia a la información obtenida. 3. Al revisar las reglas para establecer las categorías emergentes, se evidencia que al categorizar a la luz de los conceptos de la teoría existe una mejor demarcación de los códigos por utilizar; entonces, las categorías presentadas son definidas por la teoría de consecución de objetivos. 4. Una vez finalizada la categorización, se exporta al programa para establecer la conexión entre los códigos y los resultados y analizarlos en relación con la teoría para evaluar el trabajo realizado.

Finalmente, se analizó la información mediante la triangulación de datos, la cual consiste en consultar las fuentes de información, considerando distintos actores del contexto ${ }^{12}$ : en este caso, se comparó la información obtenida de la teoría de King, la entrevista, y el aporte que brindan quienes investigan, a lo que se suma el desarrollo de espacios reflexivos en donde se presentó resultados preliminares con diferentes actores que enriquecieron el proceso analítico.

\section{Consideraciones éticas}

Se tomó en cuenta varios aspectos bioéticos partiendo de la Ley N. ${ }^{\circ 9234}$-Ley Reguladora de Investigación Biomédica y de las definiciones del Informe Belmont ${ }^{13}$ (Belmont report: Ethicprinciples and rules 
fordevelopmentresearch in humans); es decir, el respeto a las personas, la beneficencia y la justicia formaron parte de los principios bioéticos del estudio, máxime considerando que en toda investigación realizada en seres humanos, es necesario contar con el consentimiento informado voluntario.

\section{RESULTADOS}

Partiendo de la teoría de King, los resultados de este estudio se evidencian y se categorizan desde los diferentes factores que pueden influir en la duración de la lactancia materna en las estudiantes universitarias que amamantan; por ende, se incluye los personales, interpersonales y los sociales, planteadas las categorías de análisis de la investigación en la teoría.

En cuanto a la categoría de los factores personales, las participantes perciben que la lactancia materna es un derecho, un beneficio, y el mejor alimento que pueden brindar a sus infantes, motivo por el que influye directamente en el mantenimiento y duración de esta, lo cual se refleja en las siguientes respuestas:

LM-P07 "Porque creo que es un derecho para ella, por los beneficios, y porque tengo el tiempo suficiente para hacerlo".

LM-P09 "La leche materna es el mejor alimento para el óptimo desarrollo del bebé, posee todos los nutrientes que requiere el niño, considero que ninguna fórmula sin importar la marca o calidad puede sustituir los beneficios que brinda la leche materna para el desarrollo integral del lactante".

Por el contrario, el disfrute o no de la lactancia materna no ha intervenido de manera significativa para que las madres tomen la decisión de abandonarla, ya que en su totalidad las participantes continuaban amamantando, lo disfrutaran o no. En este se obtuvo respuestas como:

LM-P01 "Con el segundo sí lo estoy disfrutando, con la primera no tanto porque le di poco tiempo".

LM-P05 "No, porque es un desgaste físico, aunque a veces sí soy capaz de disfrutarlo y tomarme mi tiempo".

La experiencia de ser una madre universitaria que amamanta influye de manera significativa en el proceso, ya que el tiempo, las responsabilidades académicas y el apoyo familiar e institucional con el que cuenten las madres son importantes para lograr una lactancia materna exitosa. Algunas de las respuestas de las participantes al mencionar la experiencia fueron:

LM-P01 "Es más complicado con hijos, pero es precisamente ese nivel de complicación que le da a uno más fuerzas para estudiar, antes yo estudiaba en San José y era más complicado porque no contaba con el apoyo de nadie, en cambio aquí en San Ramón hay más facilidad y apoyo".

LM-P02 "A veces es complicado, porque hay que trasladarse, salirse de clases o perder esos momentos, pero también está la parte bonita de todo eso". 
Por otro lado, las participantes perciben una poca sensibilización del proceso de lactancia materna por parte de la comunidad universitaria, lo cual influye directamente en el rol de ellas como estudiantes: al consultar si habían percibido cambios en el personal administrativo, docentes o compañeros, algunas mencionaron:

LM-P01 "Al principio nadie le dice nada a uno, pero sí me enteré de que susurraban cosas como "no hay que hacer trabajos con ella porque tiene un hijo y no cumple con los trabajos" pero luego uno les demuestra que se puede ser más responsable que muchos que no están en esta situación”.

\section{LM-P03 “De estudiantes sí, realmente hay muy poca empatía y en mi carrera eso me sorprendió”.}

LM-P04 "Realmente nunca he notado cambios o problemas para amantar; es decir, por parte de ellos no".

Además, se evidencia una necesidad de un espacio adecuado para la extracción de leche materna y para amamantar a sus infantes lo que se convertiría en un apoyo en la duración de la lactancia materna de las estudiantes madres; por ende, el tiempo y el apoyo son considerados como un elemento importante para prolongar la lactancia materna, dado que fueron seleccionados por la totalidad de las participantes. Por su parte, el empoderamiento, la motivación y conocimiento de las estudiantes madres juega un papel fundamental para la duración de la lactancia materna.

Respecto de los factores interpersonales, la familia o amigos generalmente son los que plantean la idea de abandonar la lactancia materna, lo cual se evidencia en respuestas como:

LM-P01 "Sí, porque mi mamá me dice que lo deje porque el niño no quiere comer y que se tape cuando va a la universidad para que no la vean".

LM-P04 "Sí, después del año me dicen que ya está muy grande, que se ve muy feo, que me tape, más que todo por parte de mi familia".

Sin embargo, no es un factor tan influyente para que las participantes tomen la decisión de abandonarla, máxime si cuentan con una buena comunicación y relación con la familia y amigos.

Los espacios donde la estudiante madre puede expresar sentimientos, dudas y experiencias representan un factor protector para el mantenimiento y duración de la lactancia materna, aunado a la formación o capacitación con la que cuenten en temas relacionados con la lactancia materna. En el caso de las participantes, la mayoría definió como excelente o regular su comunicación con familiares, mientras que todas habían asistido al curso de preparación para el parto.

Los resultados demuestran que las estudiantes madres lograron un equilibrio entre el rol de madre y el de estudiante, aunque no estuvieron exentas de presentar dificultades a la hora de ejercerlos.

En lo que corresponde a los resultados de la categoría de los factores sociales se encontró que el entorno familiar o social en el que viven las participantes influye directamente en la duración de la lactancia, ya que esto 
puede ayudar o dificultar el proceso, dependiendo de cómo la organización en la que se encuentran facilita, o no, la consecución de metas.

Al respecto, la Casa Infantil Universitaria, lugar donde se les brinda cuido a infantes mientras las estudiantes madres asisten a la universidad (tabla 1), se considera una fuente de apoyo fundamental para ellas, ya que este espacio es visto no solo como un lugar de cuido, sino como el único espacio para brindar lactancia materna: en caso de que no se contara con este, representaría un factor de riesgo para la duración de la lactancia materna.

Tabla 1. Universidad de Costa Rica, Sede de Occidente. Persona o Institución encargada del cuido, Recinto de San Ramón, II ciclo 2017.

\begin{tabular}{|l|c|c|c|c|c|}
\hline $\begin{array}{l}\text { Código de } \\
\text { participante }\end{array}$ & \multicolumn{2}{|c|}{ Persona o institución que cuida a su hijo o hija } \\
\hline Padre & Abuelos & Niñera & $\begin{array}{c}\text { Casa Infantil } \\
\text { Universitaria }\end{array}$ & Otro \\
\hline LM-P01 & $\mathrm{X}$ & & & $\mathrm{X}$ & \\
\hline LM-P02 & & & & $\mathrm{X}$ & $\mathrm{X}$ \\
\hline LM-P03 & $\mathrm{X}$ & & & $\mathrm{X}$ & \\
\hline LM-P04 & & $\mathrm{X}$ & & $\mathrm{X}$ & \\
\hline LM-P05 & & & & $\mathrm{X}$ & \\
\hline LM-P06 & & & & $\mathrm{X}$ & $\mathrm{X}$ \\
\hline LM-P07 & $\mathrm{X}$ & $\mathrm{X}$ & & $\mathrm{X}$ & \\
\hline LM-P08 & & $\mathrm{X}$ & & $\mathrm{X}$ & \\
\hline LM-P09 & & & $\mathrm{X}$ & & \\
\hline LM-P10 & $\mathrm{X}$ & $\mathrm{X}$ & & & \\
\hline Total & 4 & 4 & 1 & 8 & 2 \\
\hline
\end{tabular}

Fuente: Elaboración propia

De igual manera, el aspecto sociocultural influye directamente sobre las actitudes que cada madre asume al amamantar; por ende, es necesario sensibilizar a la población universitaria ya que, como se ha visto a lo largo de los resultados la lactancia materna involucra a todos por igual. En relación con dicho factor, la lactancia materna de las estudiantes universitarias, no se ha visto influida ni positiva ni negativamente por la intervención de los profesores desde la posición de autoridad que ejercen sobre ellas: en cuanto a si contaban con permisos para amamantar a sus infantes, las participantes mencionaron que,

LM-P01 "Cuando estaba más pequeño los profesores me daban permiso sin ningún problema".

LM-P02 "Sí, mi profe es muy comprensiva”.

LM-P03 “Sí, nunca me han dicho que no”. 
LM-P04 "Sí, yo salía si me llamaban de la casita infantil o así y luego volvía a clase, los profes nunca me dijeron nada".

Finalmente, no existe materia en legislación nacional que mencione, proteja o ampare a las estudiantes universitarias que se encuentran brindando no solo lactancia materna, sino en proceso de maternidad.

\section{DISCUSIÓN}

Como proceso, la lactancia involucra alimentar al infante por medio de la leche materna, el cual no solo envuelve los aspectos fisiológicos, sino que involucra el entorno en el que se desarrolla la madre, el cual influye en la duración del proceso de amamantamiento.

En el caso de las estudiantes madres que amamantan a sus hijos e hijas en la Universidad de Costa Rica, ejercen dos roles principales, el de estudiantes y el de madres: respecto de este último, la lactancia va de la mano y se convierte en lo prioritario, al punto de que puede afectar el primer rol, motivo por el que "para otros, marca un antes y un después en sus planteamientos respecto de su formación, sobre todo en aquellas que se enfrentaron al hecho tempranamente en su formación"2. Es en este nuevo rol, cuando la mujer adopta una identidad materna y unos comportamientos que favorecen la crianza del recién nacido ${ }^{14}$; por lo tanto, se puede evidenciar un cambio en las prioridades de rol, puesto que pesa más el beneficio para el niño o niña y se enfatiza el cuidado sobre el estudio.

Al ejercer el rol de madre, las participantes evidenciaron tener claro que entre los beneficios más importantes de la lactancia materna está el fortalecimiento del vínculo entre madre e hijo, además de que aporta grandes beneficios a la salud y fortalecimiento del sistema inmunológico del bebé, todo dentro de la percepción que tienen las madres sobre la necesidad de brindarle o trasmitirles beneficios a sus infantes, la cual se visualiza como una experiencia y una oportunidad única, un regalo que brindan a sus hijos para toda la vida y con el que no todos cuentan, (...) además de que también es apreciada como lo mejor para sus hijos debido a sus múltiples beneficios a nivel físico y emocional ${ }^{4}$.

La percepción se encuentra en constante cambio, construida a partir de experiencias previas, así como del aspecto cultural y crianza durante toda su vida, por lo tanto, el percibir los beneficios de la lactancia materna es resultado del conocimiento que poseen las participantes; sin embargo, existe un aspecto biológico que influye en la lactancia materna: el vínculo descrito como un instinto biológico de máxima importancia, esencial para la salud mental del infante, quienes experimentan una relación cálida, íntima y continua con la madre en la que ambos se sientan satisfechos y $\operatorname{plenos}^{15}$, lo cual refuerza la idea de prolongar la lactancia.

Otra de las razones por las cuales estas mujeres brindan lactancia materna es porque perciben que los hijos e hijas tienen derecho; no obstante, dejan de lado los beneficios para la madre: desconocen que es bueno para su salud física y mental, de ahí la importancia de que las estudiantes madres mantengan la práctica de lactancia, dados los beneficios para ambos ${ }^{16}$.

De igual forma, el tiempo y el apoyo son factores imprescindibles para mantener la práctica del amamantamiento: el primero toma relevancia en la organización y cumplimiento de metas, pues el manejo de este 
se vuelve importante a la hora de agregar otro rol a la vida cotidiana de las estudiantes madres, razón por la que desde la enfermería- se debe buscar la sensibilización desde la comunidad universitaria en el tema de lactancia materna, máxime si la empatía muchas veces es poca, ya que el no sentir el apoyo de compañeros y docentes puede llegar a dificultar la práctica de la lactancia materna.

En cuanto al tiempo, influye en duración de la lactancia materna, pues al tener que cumplir con más de un rol, el descanso de la madre está asociado a los periodos de descanso de sus hijos, a lo que se suma que el rol de estudiante universitario puede influir negativamente en prolongar el amamantamiento dado que el desgaste físico, mental y emocional aumenta considerablemente por las suma de los factores ${ }^{17}$.

En torno a la maternidad, se define como "un periodo de transición y uno de los principales acontecimientos en la vida. Es un proceso en el que se produce una interrupción del ritmo normal del día a día (...)" 14 , por lo que, de alguna manera, las madres estudiantes mencionan que "hay que priorizar entre el estudio y la lactancia": nuevamente se observa la necesidad de las participantes de mantener un equilibrio entre ambos roles en relación con tiempo, en el que se debe priorizar entre el estudio y la lactancia de acuerdo con las metas que se planteen.

Por otro lado, si se parte de que la extracción de leche es un factor protector y prolongador de la lactancia materna, con mayor razón las estudiantes deben contar con las facilidades para hacerlo en su ambiente universitario, aunque la realidad es que no tienen un espacio para amamantar y realizar la extracción de leche materna y su adecuado almacenamiento. Sin embargo, se debe rescatar que, a pesar de los pocos factores protectores, las estudiantes han logrado mantener la lactancia materna con una duración por más de un año.

Los resultados demuestran que para la duración de la lactancia materna en las madres universitarias se necesita de tiempo, espacio y facilidades que permitan cumplir con el rol de madre y el rol de estudiante, por esto "se podría decir, entonces, que para lograr un buen amamantamiento se requiere de tiempo, espacio, apoyo y facilidades" "18; por ende, si se obtiene el apoyo necesario, el uso del tiempo se vuelve más cómodo para llevar a cabo ambas actividades de estudiante y de madre. En la misma línea, es "indispensable contar con información pertinente de extracción y conservación de leche materna y un ambiente saludable que promueva la continuidad de la lactancia materna exclusiva"19. Lo anterior se relaciona con la importancia de la percepción y como esta puede ayudar a modificar dicho aspecto en cuanto a prolongar la lactancia materna.

El análisis de los factores interpersonales permitió observar que las responsabilidades de las estudiantes madres aumentan significativamente, lo cual las lleva a reorganizar y reajustar su tiempo obligatorio.

Este cambio en la vida cotidiana y de los roles se evidencia en las madres que han tenido que realizar cambios en el ejercicio de su rol, ya que de alguna manera se han tenido que ir transformando en madres que, además de brindar cuidados y suplir las necesidades fisiológicas y emocionales de su hijo o hija, también ejercen el rol de esposa, profesional, estudiante, amiga y en muchos casos suelen ser madres cabeza de familia, entre $\operatorname{otros}^{20}$.

Desde el ámbito de salud y conocimiento de profesionales en la salud, el hecho de cumplir con la meta de amamantar, repercute positiva o negativamente en el rol que se desarrolla y que está ligada esta meta. No 
obstante, al cumplir con diferentes roles, existen diferentes metas que, en el caso de las participantes, corresponden a la graduación y a su futuro profesional; es decir, van dirigidas a satisfacción personal, por lo que debe promoverse y brindar la facilidad para lograrlas, lo cual requiere la interacción de los individuos, en este caso de la comunidad universitaria, la cual tiene el rol de profesor, estudiantes, compañeros, entre otros. La importancia de estos roles es introducir la percepción de necesidad de otros de cumplir con el rol de madre simultáneo al de estudiante, lo que brindaría una ventana de concientización y de cumplimiento de objetivos: cumplir las metas de cada individuo, tanto para la madre universitaria en sus metas, como de las metas del profesor de ayudar al estudiante y del rol de estudiantes y compañeros de superarse profesionalmente.

La decisión de amamantar está fuertemente influenciada por la información acerca de la alimentación infantil que reciben las madres y por el apoyo físico y social proporcionado durante el embarazo: las actitudes familiares, médicas y culturales, las condiciones económicas, presiones comerciales y las políticas y normas, nacionales e internacionales pueden favorecer o no la lactancia ${ }^{21}$.

Otro aspecto es la comunicación, puesto que influye en relación con el estatus entre los comunicantes y el rol de estos, por consiguiente, la que se da entre la madre universitaria y su familia puede afectar más que la que se tiene con sus compañeros o profesores. Sin embargo, aun considerando lo anterior, las participantes han logrado mantener la lactancia materna, lo cual puede estar ligado a la información que la madre tenga al respecto (impartida por un profesional): un factor protector ${ }^{21}$.

Ahora bien, un factor de índole social que protege la duración de la lactancia materna es el que la madre viva con su hijo o hija, como pasa con la totalidad de las participantes de la investigación, dicho lo anterior, el núcleo familiar en el que creció y se desarrolló la madre universitaria, y el núcleo familiar en el que se desenvuelve actualmente influyen fuertemente en las metas y percepciones en relación con la duración de la lactancia materna.

En el caso de la lactancia materna, las mujeres construyen un significado partiendo de la interacción con sus familiares cercanos, así como de los significados que construyen por medio de las experiencias vividas en el proceso de lactancia materna ${ }^{22}$. Además, se determinó que el entorno familiar y social de la madre ayuda o dificulta la lactancia materna: mientras que el apoyo familiar o de pareja son fundamentales, tener una familia numerosa o estar fuera de casa son factores de riesgo ${ }^{23}$.

De igual forma, la importancia dentro del proceso de aceptación y adaptación al nuevo rol, se logra si todos los miembros del hogar redefinen su papel dentro del sistema familiar ${ }^{24}$, el cual es para las participantes una de las mayores fuentes de información e interacción, fundamentales en cuanto a tomar decisiones; no obstante, a pesar de lo mencionado, y contrario a las opiniones de sus allegados, las madres de este estudio mantuvieron la lactancia, dada su percepción del beneficio de la lactancia.

A lo largo del proceso de análisis de la información y gracias a la interacción con las madres participantes y funcionarias de la Universidad de Costa Rica, se afirma que la Casa Infantil Universitaria (CIU) es un recurso que protege la lactancia materna, ya que aunque no cuentan con un espacio destinado para practicarla, en la medida de lo posible han buscado facilitarla. Si bien es cierto que existe una organización informal dentro de la CIU, esta nace a partir de una formal que cuenta con división del trabajo, cadena de mando y una meta principal 
para lograr resultados en la organización; no obstante, conforme se dan las relaciones entre las madres y los encargados, existe una comunicación influenciada por el estatus: a raíz de esta interacción se observó necesidades de los individuos dentro de las que la percepción de las autoridades de la CIU cambia y, a la vez, modifica la organización de este espacio para brindar un lugar a las madres y lograr la metas planteadas desde el rol de madre. A lo anterior, cabe agregar que tener a los hijos en un lugar adecuado, como una guardería universitaria, brindaría más tranquilidad, además habría estabilidad emocional al saber que sus hijos e hijas están atendidos por profesionales $^{25}$. Además del cuidado de los infantes, la inversión en tiempos y recursos por el traslado de la casa a la guardería, y de ahí a la oficina o aulas y viceversa, quizá tendría como resultado menos retardos y ausentismo, mientras se resuelven problemas de apego y se apoya el cuidado, en un espacio donde hay privacidad ${ }^{26}$ y en el que el contacto piel con piel y la interacción por medio de miradas, caricias y palabras fortalecen el vínculo ${ }^{18}$ también fortalece la lactancia.

Como se mencionó, la CIU es el único lugar con el que cuenta la sede para el desarrollo de la práctica de la lactancia materna y la extracción de leche, aun cuando no fue creada para ello, ha tenido que adaptarse a las necesidades de las estudiantes, máxime que en la sede de Occidente no existe ningún espacio al respecto, factor de riesgo que puede llegar a disminuir la duración de la lactancia con las consecuencias que generaría.

Entre otros conceptos identificados en el análisis, se encuentra el de autoridad, considerada como "esencial para el logro de objetivos. Es útil para coordinar y regular conductas para alcanzar las metas. Es una manera de tratar de asegurar las expectativas del rol y el desempeño en una posición ${ }^{27}$ ". Se relaciona con la organización de la universidad, la cual tiene el poder de tomar decisiones que dirigen las actividades de uno y de los otros, por lo cual, se debe velar por aquellos factores que influyen tanto en el mantenimiento de la lactancia materna como del aspecto académico de las universitarias ${ }^{27}$.

Es a partir de esta característica que el profesional de enfermería debe ayudar a la sensibilización de los que ostentan el rol con mayor autoridad dentro de la comunidad universitaria, puesto que, dentro de sus responsabilidades se encuentra el velar por que se cumplan las metas de los estudiantes.

La universidad como organización debe velar por brindar oportunidades a sus integrantes: en el caso de las madres universitarias, la lactancia materna se trata de un derecho universal, por lo que hay que protegerla, promoverla y apoyarla -por lo menos hasta los dos años de edad ${ }^{28}$-ya que esta genera un gran impacto a un bajo costo, disminuye la morbimortalidad neonatal, infantil y materna ${ }^{29}$.

Así bien, la lactancia materna es una práctica ligada a aspectos socioculturales muy fuertes por lo que influye directamente sobre las actitudes que cada madre asume a la hora de brindar el pecho a sus hijos, como cubrir el pecho, buscar un lugar privado, o amamantar libremente donde ellas lo deseen, lo cual puede afectar positiva o negativamente la duración de la práctica de amamantamiento. Partiendo de lo mencionado, se resalta que las instituciones de educación superior tienen la responsabilidad de conocer esta realidad, desde la que no solo se trate de identificar sino también de acompañar la formación integral de sus estudiantes madres; sin embargo, en el contexto universitario, son evidentes los vacíos entre las madres estudiantes y las políticas de la universidad respecto de la maternidad, ya que la institución educativa podría — no estrictamente "debería"proporcionar algún tipo de apoyo e interesarse por su realidad ${ }^{24}$. 
En la misma línea, existe la necesidad de incentivar el interés y la investigación en la temática de lactancia materna, más allá del sector salud, dado que es un tema de interés tanto para instituciones públicas y privadas que contribuye a la salud pública del país ${ }^{30}$ : partiendo de lo mencionado, la consecución de objetivos de los individuos que forman los diferentes grupos de organización debe velar por la búsqueda de cumplimiento de metas, en este caso, de las madres universitarias que amamantan, máxime considerando que velar por la población es responsabilidad de las autoridades que tienen el poder de realizar cambios, de modo que las personas no vean disminuido su rol y su percepción, al tiempo que mejoran la relación y comunicación entre estudiantes y autoridades, con apoyo y acompañamiento en la decisión de la práctica de la lactancia materna, cuyos beneficios son tanto para las madres, hijos y familias, como para la sociedad.

\section{CONCLUSIÓN}

La lactancia materna se constituye en una estrategia de salud pública para el mejoramiento de los índices de salud infantil y materna, por lo que la promoción y el acompañamiento que como profesionales de salud se brinde a las madres durante este proceso es clave para su éxito y el de sus infantes.

Las estudiantes universitarias que son madres y que se encuentran en el proceso de amamantamiento pasan por diferentes retos para poder establecer un equilibrio entre los diferentes roles a los que se enfrentan y el cumplimiento exitoso de estos, motivo por el que las acciones que se desarrollen en conjunto dentro de la institución universitaria son fundamentales para prolongar la lactancia en esta población.

De igual forma, el éxito en la lactancia de las estudiantes universitarias madres va a estar influenciado por diferentes factores tantos personales, interpersonales y sociales que son dinámicos y que se relacionan entre sí, a los que se suma las redes de apoyo, educación en salud, acompañamiento y la acciones en pro de la lactancia materna de la institución universitaria y de sus propios entornos familiares.

Desde los factores personales se determinó que entre los aspectos más importantes que manifiestan las participantes para prolongar la duración de la lactancia se encuentran el empoderamiento, la motivación y conocimiento que ellas mismas tiene sobre el tema, así como el tiempo y el apoyo.

Respecto de los factores interpersonales, la familia o amigos generalmente son los que plantean la idea de abandonar la lactancia materna; sin embargo, al parecer no es un factor tan influyente para que las participantes tomen la decisión de abandonarla. Por otro lado, una buena comunicación y relación con la familia y amigos sí parecen estar relacionadas con prolongar la lactancia.

En lo que corresponde a los factores sociales, se determina que el entorno social en el que viven las participantes influye directamente sobre la duración de la lactancia, ya que puede ayudar o dificultar el proceso, dependiendo de cómo la organización en la que se encuentran facilita, o no, la consecución de metas.

\section{Conflicto de intereses}

Los autores declaran no tener conflicto de interés de orden personal, comercial, académico, político, financiero o de ninguna otra índole. 


\section{REFERENCIAS BIBLIOGRÁFICAS}

1) Alpízar M, Canales J, Moreira D. Factores personales, interpersonales y sociales que influyen en la duración de la lactancia materna en las estudiantes universitarias que amamantan a sus hijos e hijas en la Universidad de Costa Rica, sede de Occidente, recinto San Ramón, II semestre 2017. Alajuela, Costa Rica: Universidad de Costa Rica; 2018.

2) Castañeda M. Ser Estudiantes, madres y padres: una dualidad cotidiana. Chile: Universidad de Chile; 2015. Disponible en:

http://repositorio.uchile.cl/bitstream/handle/2250/135041/Memoria\%20de\%20Titulo\%20$\% 20$ Maternidad $\% 20 \mathrm{y} \% 20$ Paternidad $\% 20$ Universitaria $\% 20$ FINAL.pdf? sequence $=1$

3) Baeza B, Henríquez F, Prieto R. Descanso postnatal parental: experiencia en lactancia materna de madres trabajadoras usuarias del sistema público de salud en la Región de la Araucanía, de Chile. Rev. chilena nutrición. 2016; 43(2): 131-137. DOI: http://dx.doi.org/10.4067/S0717-75182016000200004

4) Bermúdez L. Vivencia de la lactancia materna desde el relato de mujeres lactantes habitante del departamento de Antioquia. Medellín, Colombia: Universidad de Antioquía; 2016. Disponible en: http://200.24.17.74:8080/jspui/bitstream/fcsh/515/3/BermudezLaura_2016_VivenciaLactanciaMaternaRel atoMujeres.pdf

5) Asociación Española de Pediatría. Lactancia materna en niños mayores o "prolongada". Madrid. España; 2015. Disponible en:

http://200.24.17.74:8080/jspui/bitstream/fcsh/515/3/BermudezLaura_2016_VivenciaLactanciaMaternaRel atoMujeres.pdf

6) OMS/UNICEF. Estrategia mundial para la alimentación del lactante y del niño pequeño. Suiza: OMS; 2003. Disponible en: https://www.who.int/nutrition/publications/gs infant feeding text spa.pdf

7) OMS/UNICEF/Red internacional de acción en materia de alimentación de lactantes. Las leyes para proteger la lactancia materna son inadecuadas en la mayoría de los países. Suiza: OMS; 2016. Disponible en: https://www.who.int/es/news-room/detail/09-05-2016-laws-to-protect-breastfeeding-inadequate-in$\underline{\text { most-countries }}$

8) Victora C, et al. Lactancia Materna en el Siglo XXI: Epidemiología, Mecanismos y Efectos a lo Largo de la Vida. Revista Lancet. 2016; 387: 475-90. Disponible en: http://www.incap.int/index.php/es/publicaciones/publicaciones-conjuntas-con-otrasinstituciones/doc_view/782-the-lancet-serie-sobre-lactancia-materna-2016-espanol 
9) Hurtado J. Metodología de Investigación. Guía para la comprensión holística de la ciencia. Cuarta Edición. Venezuela: Ediciones Quirón; 2010. Disponible en: https://es.scribd.com/doc/312670255/JHurtado-de-Barrera-Metodologia-de-Investigacion-Revisado

10) Hernández R, Fernández C, Baptista P. Metodología de la investigación. Sexta Edición. México D.F.: McGraw Hill Interamericana; 2014.

11) Cisterna, F. Categorización y triangulación como procesos de validación del conocimiento en investigación cualitativa. Theoria. 2005; $14 \quad$ (1): 61-71. Disponible en: http://www.redalyc.org/html/299/29900107/

12) Barrantes R. Investigación: un camino al conocimiento, un enfoque cualitativo, cuantitativo y mixto. San José: EUNED; 2014.

13) República de Costa Rica. Asamblea Legislativa. Ley N. 9234 Reguladora de Investigación Biomédica. Diario Oficial La Gaceta (79). 2014.

14) Belintxon-Martín M, Zaragüeta M, Adrián M, López-Dicastillo O. El comienzo de la lactancia: experiencias de madres primerizas. Anales Sis San Navarra. 2011; 34(3): 409-418. DOI: http://10.4321/S1137-66272011000300007

15) Olza I, Marín M. Neurobiología del vínculo maternofilial: aplicaciones para la lactancia materna y/o artificial. En AEPap ed. Curso de Actualización Pediatría. Madrid: Exlibiris Ediciones; 2014. $29-39$.

Disponible en: http://cursosaepap.exlibrisediciones.com/files/49-239-

fichero/Neurobiolog $\% \mathrm{C} 3 \% \mathrm{ADa} \% 20 \mathrm{del} \% 20 \mathrm{v} \% \mathrm{C} 3 \% \mathrm{ADnculo.pdf}$

16) Lozano de la Torre M. Beneficios de la lactancia materna. España; 2006. Disponible en: https://www.aeped.es/sites/default/files/documentos/lm.pdf

17) Estivill E, Albares J, Segarra F, Roure N, Pascual M, Estivill C. Hábitos adecuados de sueño compatibles con lactancia materna a demanda. Pediatría Atención Primaria. 2008; 10(38): 15-24. Disponible en: http://www.redalyc.org/articulo.oa?id=366638702002

18) Pinilla E, Domínguez C, García A. Madres adolescentes, un reto frente a los factores que influyen en la lactancia materna exclusiva. Revista Enfermería Global. 2014; 13(33): 59-70. Disponible en: http://scielo.isciii.es/pdf/eg/v13n33/clinica4.pdf

19) Campoverde Z, Chocho M. Estrategia educativa en lactancia materna para adolescentes embarazadas que asisten al centro de salud número 1 Pumapungo Cuenca, 2014. Cuenca: Universidad de Cuenca; 2014. Disponible en: http://dspace.ucuenca.edu.ec/bitstream/123456789/20564/1/Tesis\%20de\%20Pregrado.pdf 
20) Ospina J, Urrego A, Betancourt E. La importancia de la lactancia materna en el desarrollo físico, psíquico y relacional del niño. Revista do NESM. 2015; 12(1): 7-18. Disponible en: http://pepsic.bvsalud.org/pdf/vinculo/v12n1/v12n1a03.pdf

21) Calvo C. Factores socioeconómicos, culturales y asociados al sistema de salud que influyen en el amamantamiento. Rev. Enfermería Actual en Costa Rica. 2009; (15): 1-8. Disponible en: www.revenf.ucr.ac.cr/factoreslactancia.pdf

22) Teixeira M, Argolo N, Rosane G, De Gasperi P y Siedler M. Significados de avós sobre a prática do aleitamento materno no cotidiano familiar: a cultura do querer-poder amamentar. Revista Texto \& Contexto - Enfermagem. 2016; 15(1): 98-106. DOI: https://dx.doi.org/10.1590/S010407072006000100012

23) Becerra F, Rocha L, Fonseca D, Bermúdez L. El entorno familiar y social de la madre como factor que promueve o dificulta la lactancia materna. Revista Facultad Médica. 2015; 63(2): 217-227. DOI: http://dx.doi.org/10.15446/revfacmed.v63n2.44051

24) Aponte MR, Correa D. Calidad de vida de madres adolescentes estudiantes Universitarias. Rev. Colomb. Psiquiatr. 2012; 41(3): 536-549. Disponible en:

http://www.scielo.org.co/scielo.php?script=sci_arttext\&pid=S003474502012000300006\&lng=en\&tlng=es.

25) Ávila AL. Magaña AB, Becerra E. Universidad pública y crianza de infantes. Reflexiones sobre su conciliación en la universidad de guadalajara. Ra Ximhai. 2016; 12(1): 83-104. Disponible en: http://www.redalyc.org/articulo.oa?id=46146696006

26) Sipsma H, Magriples U, Divney A, Gordon D, Gabzdyl E, Kershaw T. Breastfeeding Behavior Among Adolescents: Initiation, Duration, and Exclusivity. Journal of Adolescent Health. 2013; 53(3): 394-400. DOI: https://doi.org/10.1016/j.jadohealth.2013.04.005

27) King I. Enfermería como profesión. México: Editorial LIMUSA; 1984.

28) Estado Plurinacional de Bolivia. Seguridad Social. Reglamento a la Ley No3460 De fomento a la lactancia materna y comercialización de sus sucedáneos. Decreto supremo No0115/ 06. 2009.

29) Mercedes E. Normativa legal de protección a la lactancia materna en Venezuela. Archivos Venezolanos de Puericultura y Pediatría. 2013; 76(4): 162-168. Disponible en: http://www.scielo.org.ve/scielo.php?script=sci_arttext\&pid=S0004-06492013000400006

30) Massó E. Lactancia materna y revolución, o la teta como insumisión biocultural: calostro, cuerpo y cuidado. Dilemata. 2013; (11): 169-206. Disponible en: http://www.dilemata.net/revista/index.php/dilemata/article/view/198/239 